\title{
Force-Directed Tracking in Wireless Networks using Signal Strength and Step Recognition
}

\author{
Markus Völker \\ and Dorothea Wagner \\ Institute for Theoretical Informatics (ITI) \\ Karlsruhe Institute of Technology (KIT) \\ Karlsruhe, Germany \\ \{markus.voelker, dorothea.wagner\}@kit.edu
}

\author{
Johannes Schmid, Tobias Gädeke, \\ and Klaus Müller-Glaser \\ Institut für Technik der Informationsverarbeitung (ITIV) \\ Karlsruhe Institute of Technology (KIT) \\ Karlsruhe, Germany \\ \{johannes.schmid, tobias.gaedeke, klaus.mueller-glaser\}@kit.edu
}

\begin{abstract}
Force-directed approaches, also known as spring embedders, are widely used in the context of graph drawing and network embedding. In this paper, we study the application of these methods to signal-strength based tracking in wireless networks. The performance of the presented algorithms is evaluated based on pedestrian tracking experiments in a 60-node wireless sensor network (WSN). Additionally, we compare the outlined approach with implementations of Extended Kalman Filters (EKF) and examine similarities and distinctions between both approaches.

The algorithms are developed in a 3-step process. First, we take a brief look at the trilateration problem, where a position is estimated based on a set of noisy signal strength measurements. From this we conclude how signal strengths can be translated into adequate spring forces. Subsequently, we establish a movement model by introducing additional forces between consecutive position estimates. Finally, we show how step information from a pedestrian can be used to improve the localization.

Our experimental results indicate that force-directed methods offer an interesting and competitive approach to the tracking problem. Especially the possibility to easily include further information by introducing additional forces makes them very attractive. As modeled forces are not limited to linear functions, non-linear aspects such as distance estimates can be effortlessly modeled. We conclude that the application of force-directed approaches to the tracking problem offers a worthwhile direction for future research.
\end{abstract}

\section{INTRODUCTION}

The localization of devices has been a topic of great interest ever since the upcoming of wireless sensor network technology around the end of the last century. Correlating a measured value with its spatial position is critical to most sensing applications [1]. Within this context, location tracking can be described as the problem to determine the location of a mobile subset of sensor devices in relation to others or a reference coordinate system. This tracking can be achieved based on various input data sources. A lot of previous work in this field originates from the robotics community where the tracking of mobile robots based on angles or distances in respect to (fixed) landmarks has been evaluated thoroughly, e.g. [2]. Methods in this field especially include Bayesian information processing approaches that maximize the likelihood of a certain location under the assumption of underlying probability distributions of the available measurements [3].
In this paper, we present a force-directed approach to the location tracking problem. Force-directed approaches are frequently used in the areas of graph drawing and network embedding. The idea is to represent network nodes as steel rings and distances between these nodes as springs. The resulting spring network is usually under tension, with some distances being shorter or longer than intended. An embedding is attained by iteratively moving nodes until all forces of the network are in a state of equilibrium.

The approach to the tracking problem considered in this paper works very similar: landmarks (devices with known positions) in the WSN are represented by anchor nodes in the graph-theoretic network. Measurement positions of the mobile device in the WSN, i.e. positions at which distance measurements to landmarks are taken, are represented by position nodes in the graph. The (inaccurately) measured distances are represented by springs (cf. Figure 1 right). An estimate for the position of the mobile device is then found by iteratively moving the position node in the graph so that an equilibrium state of all spring forces is reached.

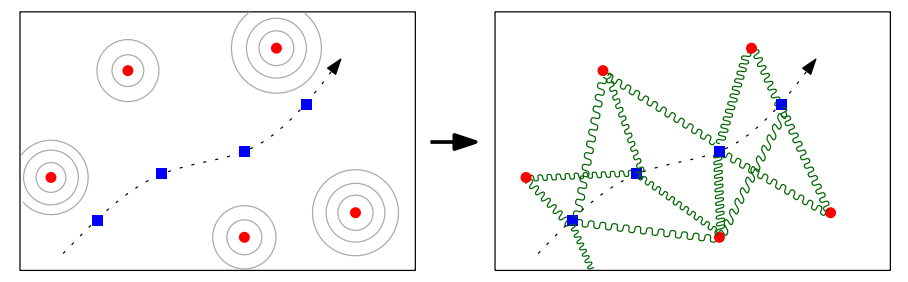

Fig. 1. Distance measurements are translated into springs. Position estimates are computed by finding force-equilibria. Left: real-world sensor network. Circles correspond to landmarks, squares to the positions of the mobile device at which distance measurements to landmarks are taken. Right: spring graph. Each landmark is represented by an anchor node, each measurement position by a position node. Distance measurements are represented by springs.

As an example application, we consider the tracking of a person carrying a mobile device. The device obtains received signal strength (RSS) values of radio packets sent by landmarks. From these RSS values, distance estimates are inferred. Based on the described representation as nodes and springs in a network, an estimation of the position of the mobile device is computed. In this paper, we compare the performance of 
force-directed tracking to a similar model in an Extended Kalman Filter (EKF) and show similarities and distinctions between the approaches. As most of today's mobile devices are equipped with an acceleration sensor, we also outline the inclusion of acceleration-based step recognition and show how the achievable accuracy is improved.

The performance of the developed force-directed tracking algorithm and the EKF are evaluated on experimental data from a 60-device wireless sensor network (WSN) deployed in two different buildings.

The key contribution of this work is to outline and experimentally evaluate the possibilities of using force-directed approaches for tracking. In comparing resulting tracking accuracies to the EKF, a qualitative assessment is provided. The experimental evaluation also studies the influence of algorithm parameters and number of used landmarks.

\section{RELATED WORK}

Most outdoor location discovery and tracking problems have been solved with the upcoming of global navigation satellite systems (GNSS) such as GPS or others. However, especially in indoor scenarios, these problems still pose a considerable challenge. In this section of the paper, we outline some relevant approaches to tracking in wireless sensor networks (WSN) and lead over to related graph-embedding problems.

\section{A. RSS-based location tracking in sensor networks}

Various approaches to track mobile sensor devices in WSN have been proposed. In the Motetrack system, locations are correlated with landmarks within range (RF-fingerprinting) by means of a calibration procedure. Based on a probabilistic approach, the mobile device can then estimate its position from these reference positions given the set of landmarks within its range. For this, it is assumed that the landmarks periodically broadcast their location [4]. Different other techniques to determine a location from such fingerprint databases as well as different application scenarios have been presented [5], [6].

However, the common disadvantage of these systems is the required survey procedure to obtain this database initially. Although crowd-sourcing is a possible solution to this [6], the presence of such a database can usually not be assumed.

An alternative approach is to infer distances from RSS values and then use trilateration to obtain a position estimate [7]. The distance estimates obtained from this method are however subject to strong fluctuations, especially in indoor scenarios [8]. As smoothing over time can usually not be applied if the receiver is moving, other methods to cope with this have been presented. One possibility are Bayesian information processing methods like Kalman filters [9], [10] . Also the inclusion of inertial data from gyro- or acceleration sensors can help to solve this issue [11].

\section{B. Force-directed approaches for localization}

Using force-directed methods to find embeddings for networks in the 2-dimensional Euclidean space is a fairly common approach. Usually, the first step is to find an initial embedding from multidimensional scaling techniques [12]. This initial solution is then locally refined by modeling attracting or dispersing forces between the nodes of the network. One application of a comparable method to the localization problem in WSN has been proposed in [13]. In this work the authors use such a force-based approach in a refinement step after an initial cluster localization. Another evaluation with a particular focus on larger networks is presented in [14]. The authors compare several force-directed approaches to find embeddings of sensor networks based on signal strengths and angular information. In [15], a distributed force-directed algorithm that computes a network embedding based on local distance and angular information is introduced.

However, most previous work is mainly evaluated in simulations and with a focus on theoretical aspects. An application to the tracking problem has not yet been studied.

\section{FORCE-DIRECTED TRACKING}

\section{A. Basic Approach}

Basically, all applications of force-directed methods to compute embeddings of graphs and networks rely on the same model: distance estimates between graph nodes are modeled as virtual springs, and an embedding is found by moving nodes until an equilibrium of forces is established [16], [17].

As outlined, we introduce two types of nodes: anchor nodes corresponding to landmarks with known positions and position nodes corresponding to the position of the mobile device at the time of measurements. The positions of the anchor nodes in the spring network are fixed, position nodes are moved based on applied spring forces that can result from underlying model assumptions or various kinds of measurements.

The lengths of the springs are defined such that either an attracting force is exerted if the position node is farther away from the anchor node than expected or a repelling force otherwise. The position estimate for the mobile device can be inferred from the most recent position node as soon as an equilibrium of forces is reached. In comparison to the graph embedding problem where an embedding for the whole network is to be found, the situation is thus simpler, as in each time step only a single node is embedded. An important aspect of the force-directed approach is that it makes almost no restrictions on the mathematical representation of the modeled forces. This allows designing forces that are highly adjusted to the characteristics of the underlying measurements.

\section{B. Forces from Distance Measurements}

The most obvious spring forces are inferred from (inaccurate) distance measurements. These distance measurements can, e.g., result from a conversion of RSS values from received radio packets as outlined in Section VI-B. Each measured distance is compared to the corresponding distance between the current position node and the respective anchor node and results in a spring. This spring either exerts a repelling force if the measured distance is bigger then the distance between the two nodes, or an attracting force otherwise. If multiple measurements are available, multiple springs exert forces in 
different directions. To reach equilibrium, the position node now has to be moved in the direction of the vector resulting from adding up all force vectors. In the following, we discuss three different possibilities for the definition of forces.

Let $d_{a, k}$ be the distance estimate to anchor node $a$ at time $k$, $\vec{v}_{a}=\left(x_{a}-x_{k}, y_{a}-y_{k}\right)^{T}$ the vector pointing from the current position estimate $\left(x_{k}, y_{k}\right)$ to position $\left(x_{a}, y_{a}\right)$ of anchor $a$, and $\left|\vec{v}_{a}\right|$ the Euclidean length of $\vec{v}_{a}$. The first force $\vec{F}_{1}$ considered in our study is defined as follows:

$$
\vec{F}_{1}=\frac{\vec{v}_{a}}{\left|\vec{v}_{a}\right|} \cdot d_{a, k}-\vec{v}_{a}
$$

This definition corresponds to the desired behavior: if the estimated distance is larger than the current distance, then $F_{1}$ points in the direction of the anchor node, otherwise it points in the opposite direction. The length of the force vector is proportional to the absolute difference between the current distance and the estimated distance.

The second force, $\vec{F}_{2}$, uses an additional factor of $d_{a, k}^{-1}$ to weaken the influence of measurements over long distances:

$$
\vec{F}_{2}=\frac{\vec{F}_{1}}{d_{a, k}}
$$

This definition takes into account that the average distance estimation error increases more or less linearly with the sender-receiver distance. By dividing through the estimated distance, more inaccurate measurements from longer distances are weakened so that they do not worsen the position estimate.

The third force definition is motivated by the graph drawing algorithm of Fruchterman and Reingold [17], which is known to work very well for the computation of graph embeddings, and which has also been used to compute embeddings of wireless sensor networks [14]. For each range measurement an attractive force $\vec{F}_{\text {attr }}$ and a repulsive force $\vec{F}_{\text {rep }}$ are introduced as follows:

$$
\vec{F}_{\text {attr }}=-\frac{\left|\vec{v}_{a}\right|^{2}}{d_{a, k}} \cdot \frac{\vec{v}_{a}}{\left|\vec{v}_{a}\right|}, \quad \vec{F}_{\text {rep }}=\frac{d_{a, k}^{2}}{\left|\vec{v}_{a}\right|} \cdot \frac{\vec{v}_{a}}{\left|\vec{v}_{a}\right|} .
$$

The acting force $\vec{F}_{3}$ is then given by $\vec{F}_{3}=\vec{F}_{\text {attr }}+\vec{F}_{\text {rep }}$.

Each of the three described force definitions could be used to model RSS-based distance estimates in a force-directed approach. In Section VI-C we compare the three possibilities. According to the experimental study, $\vec{F}_{2}$ has been found to be the most promising force for representing RSS measurements.

\section{Forces from Movement Model Assumptions}

Due to the high fluctuations in RSS-based distance estimations, the position estimate can jump in the range of meters, even for a stationary receiver. To avoid this, we use the fact that the time difference between consecutive measurements depends on the measurement update rate. This is used as additional system knowledge (in our experiments: 4 measurements per second for each landmark). Assuming a maximum velocity $v_{\max }$ of the tracked object, a maximum distance $\Delta t \cdot v_{\max }$ is assumed for the movement of the receiver during one time period $\Delta t$. To model this, we introduce additional spring forces between consecutive position nodes (cf. Figure 2). For example, a pedestrian usually does not

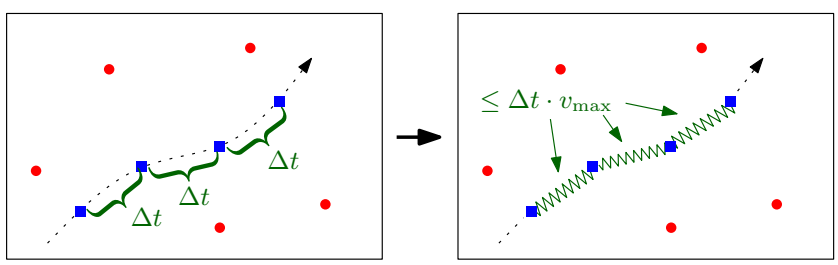

Fig. 2. Time differences are translated into distance estimates.

move faster than $v_{\max }=5 \mathrm{~km} / \mathrm{h}$. From this we can infer that consecutive measurement positions should be no further apart than $d_{\max }=\Delta t \cdot v_{\max }$, with $\Delta t$ being the time difference between two measurements.

Let $\left(x_{k-1}, y_{k-1}\right)$ be the final position estimate for time step $k-1$ and $\vec{w}=\left(x_{k-1}-x_{k}, y_{k-1}-y_{k}\right)^{T}$ the vector pointing from the current position estimate at time $k$ to the position estimate at time $k-1$. By introducing the force

$$
\vec{F}_{\text {move }}=\left\{\begin{array}{ll}
\overrightarrow{0} & \text { if }|\vec{w}| \leq d_{\max } \\
\left(|\vec{w}|-d_{\max }\right) \cdot \vec{w} /|\vec{w}| & \text { if }|\vec{w}|>d_{\max }
\end{array},\right.
$$

we model an attracting force that acts on the position node as soon as it is located more than distance $d_{\max }$ away from the previous position node. This force is proportional to the amount distance $d_{\max }$ is exceeded. If the position nodes are closer than $d_{\max }$, no force is introduced. This example shows how easily non-trivial forces can be represented.

Note that there is no need to store more than two position nodes at the same time, the current position node and the position node representing the last position estimate.

\section{Forces from Step Recognition}

Today, most mobile devices have a built-in accelerometer, which can be used to identify steps by recognizing peaks in the acceleration pattern. As an example of how to extend the system model with additional information, we study the possibility of incorporating such step information. As we do not want to go into the details of step detection, we just assume knowledge of occurring steps for the purpose of this paper.

So far we assumed that the position is re-estimated every time when new measurements arrive. With each new set of measurements, a new position node was introduced and the old position node was removed from the network. If step information is available, we use a slightly different approach. Now new position nodes are only introduced if steps are detected. If new distance measurements are obtained, the corresponding forces are simply attached to the current position node. Again, a connection of the current step node with the previous step node is incorporated by an additional force similar to $\vec{F}_{\text {move }}$ from the last paragraph (cf. Figure 3)

There are two differences to the model without step recognition: $d_{\max }$ is now set to an average step distance and, as we now know that the person actually moved, one can also make the force repelling if the distance between the position nodes is shorter than a usual step. 


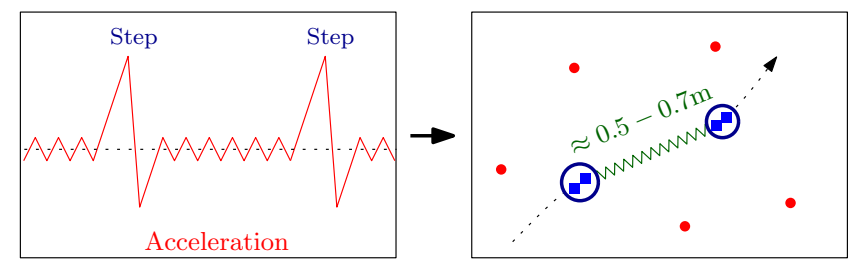

Fig. 3. Step detection is used to detect and combine co-located measurements.

Of course, if the person does not move for a while, it can happen that many measurements accumulate between two steps. In this case, it is usually sufficient to use only the $k$ latest measurements, with $k$ being chosen appropriately for the considered application.

\section{E. Approximating the State of Equilibrium}

After determining all forces that are exerted by the introduced springs, a state of equilibrium, i.e. a local optimum, has to be found. In this paper, all forces are considered to be 2dimensional vectors. For each kind of force, we define an additional weighting that allows controlling the importance of the forces relative to each other. By adding the vectors multiplied by the corresponding weight, we get the direction to which to move the position estimate. The movement is done in small increments of $5 \mathrm{~cm}$ to $10 \mathrm{~cm}$. After each movement, the acting forces are recomputed to get the new movement direction. The number of necessary iterations depends on the scenario. For tracking without step detection we use 7 iterations with $10 \mathrm{~cm}$ movements, followed by 3 iterations with $5 \mathrm{~cm}$ movements. In the model with step detection, we recompute the position only once per step. Accordingly, we use a slightly higher number of iterations: 12 iterations with $10 \mathrm{~cm}$ movements followed by 3 iterations with $5 \mathrm{~cm}$ movements. More dynamic position changes could be enabled by greater displacement distances or a higher number of iterations. Of course, iterations could also be done as long as an improvement is achieved and interrupted otherwise.

\section{F. Time and Space Complexity}

Both time and space demand of the force-directed approach are very low. For landmarks and position nodes, only coordinates have to be stored. At no point in time more than two position nodes have to be kept in memory-one for the current position estimate and one for the last position estimate, to allow the usage of movement forces. In each iteration of a measurement update, the movement direction is determined by simply adding up some 2-dimensional force vectors. This can be done very efficiently in time $O(f)$, with $f$ being the number of forces. As the number of iterations is fixed (10-15 in our experiments), the complexity of the whole measurement update also is in $O(f)$. Summarizing, both time and space complexity of measurement updates are linear in the number of involved forces. This makes the force-directed approach very attractive even for application on devices with very low computational power or little memory.

\section{EXtended Kalman Filter (EKF)}

As Kalman filters are often considered for the location tracking problem, we use an EKF as reference approach to the force-directed tracking and highlight similarities and distinctions between both. For the EKF, we keep the description to a minimum and refer to the standard literature, e.g., [18]. The descriptions and implementations of our EKFs are based on the tutorial on Kalman filters by Welsh and Bishop [19].

\section{A. EKF with Movement Model}

Similar to the model established in Section III-C, we use the knowledge that a person can only move a limited distance between two consecutive measurements. The process state at time step $k$ consists of the two-dimensional coordinates $\left(x_{k}, y_{k}\right)$ and movement is introduce in form of process noise. This results in the following process model:

$$
\vec{x}_{k}=\left(\begin{array}{c}
x_{k} \\
y_{k}
\end{array}\right)=\left(\begin{array}{c}
x_{k-1}+w_{1} \\
y_{k-1}+w_{2}
\end{array}\right)
$$

The noise terms $w_{1}$ and $w_{2}$ are assumed to be normally distributed and zero-mean. The measurement $\vec{z}_{k}$ at time step $k$ is given by

$$
\vec{z}_{k}=\left(\begin{array}{c}
\sqrt{\left(x_{k}-x_{a_{1}}\right)^{2}+\left(y_{k}-y_{a_{1}}\right)^{2}}+v_{1} \\
\vdots \\
\sqrt{\left(x_{k}-x_{a_{m}}\right)^{2}+\left(y_{k}-y_{a_{m}}\right)^{2}}+v_{m}
\end{array}\right),
$$

where $\left(x_{a_{i}}, y_{a_{i}}\right)$ is the position of the $i$-th landmark used at time $k$ and $v_{i}$ is the measurement noise for the distance estimation to this landmark. If not stated otherwise, we use the $m=10$ measurements with highest RSS, as this offers a good compromise between computational effort and achievable accuracy. The variance of $v_{i}$ depends on the RSS, as distance estimations based on weak signals involve higher uncertainties than distance estimations based on strong signals. We inferred the uncertainties of the distance estimations directly from a set of training data. The parameters used for the evaluation are stated in the experimental section VI.

\section{B. EKF with Step Detection}

Like in Section III-D for the force-directed approach, the EKF can also be extended with step information in the system model. We now separate process updates and measurement updates. While measurement updates still occur every time when new RSS measurements arrive, process updates are only performed when a new step has been detected. Additionally, the variance of the process noise in equation (5) is adjusted to reflect the expected position change during a step.

Another possibility to improve the EKF would be to use a more complex system model, e.g. a position-velocity model, by including additional variables (e.g. heading) in the state vector. However, we know from previous work that the outcome only changes marginally if high update rates are used but that the computational effort increases drastically. Given the high update rate of $4 \mathrm{~Hz}$ for the undergone experimental study we thus decided to refrain from using a more complex model. 


\section{COMPARISON OF ForCE-DiRECTED TRACKING AND EKF TRACKING}

Overall, the modeling of the force-directed approach and the described EKF is done in a similar way. However, there are several distinctions: Concerning the measurements, the EKF uses a linearization around the current position estimate, whereas the force-directed approach can model the distances directly, using non-linear terms.

Both the force-directed approach and the EKF combine a measurement with a prediction from a movement model. In the EKF, prediction and measurement update are initially computed separately and then fused based on the covariance estimates that describe the uncertainties of prediction and measurement. In contrast, in the force-directed approach both measurement and movement forces are considered concurrently. The position estimate is computed by finding an equilibrium of forces for all kinds of forces at the same time.

As there is no directional information available, the EKF uses a normal distribution with mean at the last position as prediction for the next time step. This means that the last position is the most likely one and the likelihood that the mobile device is in a certain position decreases with distance to the last position estimate. In the force-directed approach, the movement force only acts if the distance to the previous position estimate exceeds some distance $d_{\max }$. This means that all positions within distance $d_{\max }$ are equally likely with respect to the movement model. If step information is used, the EKF still assumes the last estimate to be the most likely position at the current time step, but with an increased uncertainty. In the force-directed approach, an attractive force is acting if the positions are too far apart and an additional repulsive force if they are too close together.

\section{EXPERIMENTS}

\section{A. Experimental Setup}

We deployed a WSN with 60 nodes in two different office buildings (cf. Figures 4 and 9). The devices were programmed

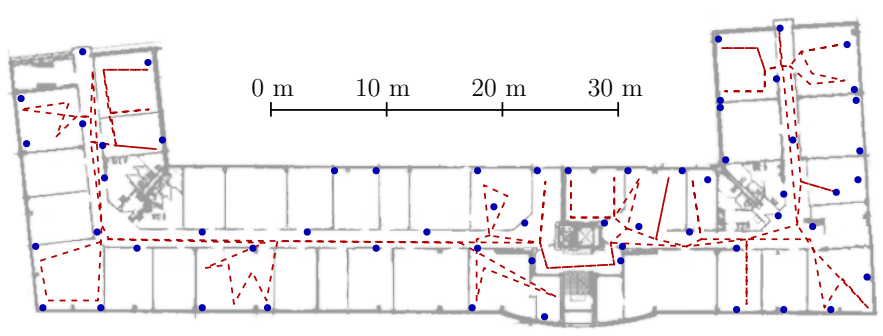

Fig. 4. Building 2: floor plan with positions of the 60 landmark devices (disks) and one sample trajectory (dashed line).

to broadcast their positions at $4 \mathrm{~Hz}$ (landmarks with known positions). Data were collected by a person walking through the network on varying predefined trajectories and carrying one sensor in front of the chest attached to a lanyard. Additionally, we recorded acceleration data in a belt bag on the hip of the user simultaneously. This data was used to perform step recognition in the presented localization approaches. A corresponding reference trajectory was established by manually determining timestamps and corresponding positions on the trajectory. Based on these points, a linear interpolation was applied to retrospectively determine the position of the pedestrian at every time step. Table I gives some statistical information on the nine experimental runs used in this paper.

\begin{tabular}{|l|c|c|c|c|c|c|c|c|c|}
\hline \multirow{2}{*}{ Scenario } & \multicolumn{3}{|c|}{ Building 1} & \multicolumn{5}{c|}{ Building 2 } \\
\cline { 2 - 10 } & 1 & 2 & 3 & 4 & 1 & 2 & 3 & 4 & 5 \\
\hline Duration (s) & 408 & 334 & 104 & 325 & 289 & 681 & 658 & 910 & 206 \\
Walked Distance (m) & 198 & 214 & 87 & 261 & 257 & 460 & 461 & 615 & 218 \\
Received Packets & $38 \mathrm{k}$ & $31 \mathrm{k}$ & $9 \mathrm{k}$ & $30 \mathrm{k}$ & $34 \mathrm{k}$ & $75 \mathrm{k}$ & $70 \mathrm{k}$ & $99 \mathrm{k}$ & $24 \mathrm{k}$ \\
Detected Steps & 395 & 424 & 155 & 497 & 443 & 879 & 890 & 1157 & 346 \\
\hline
\end{tabular}

TABLE I

PRoperties OF THE EXPERIMENTAL RUNS

\section{B. Mapping Signal Strengths to Distances}

Various approaches to infer distances from RSS values have been proposed, e.g., [20], [21]. Scenarios within buildings, as considered in this paper, are often approached by means of the log-distance path-loss model [10]. In this paper, we use a direct mapping from RSS values to the expected distances. For the calibration procedure we use an additional training data set consisting of about 300, 000 RSS-distance value pairs. This data set was determined with the same experimental setup as outlined in Section VI-A, but in additional runs. The RSS-distance-mapping was computed by determining the mean signal strength per distance. Similarly, we determined the variance of the measurement noise from this distribution to use it in the Kalman filter.

\section{Comparison of Force Definitions: Trilateration}

For both EKF and force-directed approach, the key to good tracking results lies in the combination of movement model and measurements. Nevertheless, in this section we are going to reduce both approaches to their measurement part. The reason is that we want to study the effects of different measurement force definitions without having to care for interfering effects caused by the fusion of knowledge from previous time steps. This leads us directly to the trilateration problem, where we are given a set of measurements which have been received at the same position, and the goal is to compute a good position estimate. Each measurement (consisting of multiple RSS values from different landmarks within one time step) defines a separate trilateration problem, thus resulting in several thousand trilateration instances.

To adjust the force-directed approach to the trilateration problem, we ignore the movement forces between consecutive position nodes. Using one of the forces described in Section III-B, the position estimate is now determined as follows: at the beginning, the position estimate is initialized to the position of the landmark from which the strongest signals are received. Subsequently, we use 40 improvement steps with displacement distance $20 \mathrm{~cm}$, followed by 5 steps with distance $5 \mathrm{~cm}$. The reason for the high number of iterations used for 
the trilateration is that we now cannot expect that the initial position estimate is close to the true position.

To evaluate how the measurement update of the EKF behaves in comparison to the force-directed approach, we try to analyze it isolated from the prediction. However, due to the strongly intertwined prediction and measurement in the EKF, this is not easily possible. For instance, the EKF uses a linearization of the measurement function around the current position estimate to approximate the non-linear system with a linear one. We decided to initialize the EKF with the true position (according to the reference path) as prediction and then analyzed how the measurement update distorts this ideal position estimate. To minimize the influence of the prediction, we set the corresponding covariance extremely high so that in the final result only the measurement result is weighted.

In the following study, the force-directed approaches using $\vec{F}_{1}, \vec{F}_{2}$, and $\vec{F}_{3}$ are denoted Force $F_{1}$, Force $F_{2}$, and Force $F_{3}$, respectively. The results based on the EKF measurement update are labeled EKF (Meas.). For all approaches the corresponding parameters are chosen such that only the effects of the measurement are considered.

1) Influence of Number of Used Measurements: The first study considers the influence of the number of used measurements. Figure 5 shows the average distance between the estimated position and the reference position for different counts of used measurements. Each time, only the measurements with highest signal strengths were used. Apparently,

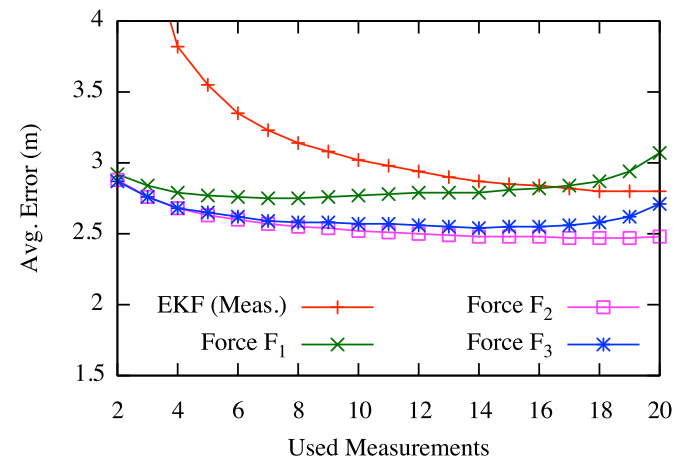

Fig. 5. Trilateration: influence of number of used measurements.

when the forces $\vec{F}_{1}$ and $\vec{F}_{3}$ are used, the results get worse with increasing number of used measurements. The reason is that both approaches try to optimize the absolute deviation from the estimated distance. As the average ranging error grows with distance, more inaccurate measurements over long distances have a stronger influence than better measurements over short distances. By using force $\vec{F}_{2}$ instead, this problem is successfully avoided. The EKF measurement update resulted in a higher avg. error, declining with more measurements used. Even though the true position was used for the EKF prediction, the error for small numbers of used measurements is notable.

2) Influence of Number of Landmarks: Figure 6 shows how the trilateration precision depends on the number of landmarks. In order to select the used landmarks, we used the following procedure: first, we selected the two landmarks with maximum distance from each other. Subsequently, we iteratively chose the landmark that maximizes the minimum distance to any already selected landmark until the intended number of landmarks was reached. For each trilateration instance, the 10 best RSS measurements were used. The results in Figure 6 show

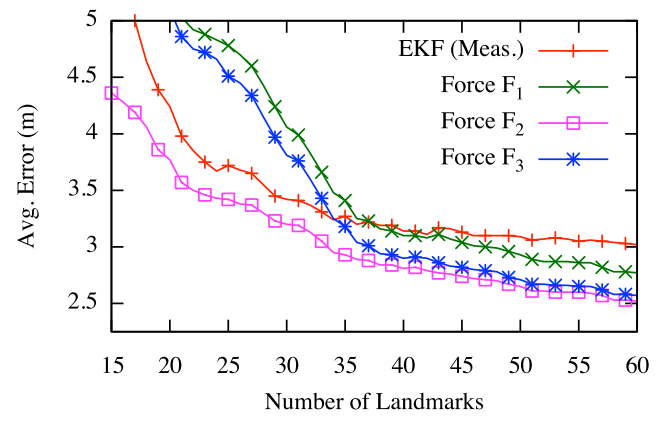

Fig. 6. Trilateration: influence of number of landmarks.

that all approaches benefit from a higher number of landmarks. In networks with less than 35 nodes, even weak signals find their way into the top ten RSS measurements. This explains the bad performance of forces $\vec{F}_{1}$ and $\vec{F}_{3}$ in sparse networks. For these forces, using only the five best measurements would actually improve the localization accuracy. Again, the force $\vec{F}_{2}$ shows the best performance.

\section{Tracking}

Tracking, i.e., the continuous localization of a moving object, could in principle be achieved by applying trilateration to each set of measurements. However, without a movement model the position estimates jump significantly due to the high fluctuation of RSS measurements. In this section, we examine the effects of the movement models for the forcedirected approaches and the EKF.

1) Parameter Influence: In the considered EKF model, movement is modeled by process noise. The higher the uncertainty of the process state, the stronger is the influence of measurement updates on the final position estimate. Figure 7 (left) shows how the overall localization accuracy of our EKF implementations, averaged over all nine experiments, depends on the assumed standard deviation $\sigma_{w}$ of $w_{1}$ and $w_{2}$ (cf. Equation 5). We first analyze the extreme cases. If $\sigma_{w}$ is chosen very low, measurement updates have little influence and the position estimate cannot keep up with the movement of the tracked object. In this case, the localization error can become arbitrarily high. If $\sigma_{w}$ is chosen too high, the prediction loses influence and the position estimate is mainly determined by the measurement update. This situation is similar to the one analyzed in the trilateration section.

For our experiments, the optimum values for $\sigma_{w}$ are $7.5 \mathrm{~cm}$ for the model without step detection and $11.5 \mathrm{~cm}$ for the model with step detection. We will use this parameters for the following analysis. However, one has to keep in mind that good values for $\sigma_{w}$ strongly depend on factors such as walking velocity or the uncertainties of the measurements. Under 

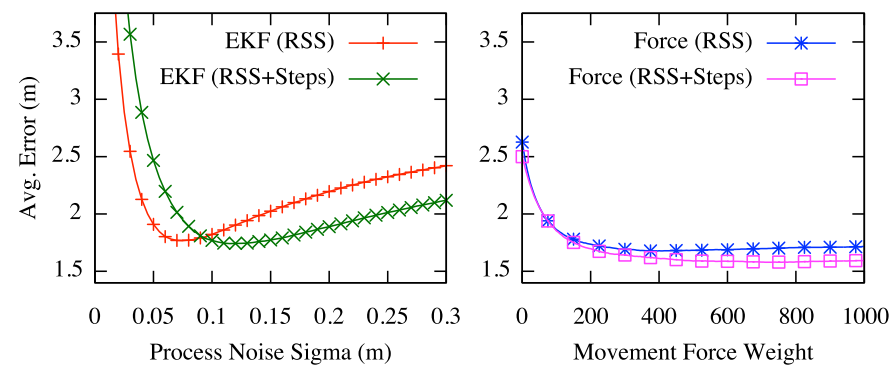

Fig. 7. Influence of model parameters on the localization accuracy. Left: influence of process noise in the Kalman filter. Right: influence of movement force weighting in the force-directed approach.

realistic circumstances, these factors are not fully known, so one will usually not be able to use optimum parameters.

In the outlined force-directed approach, the behavior of the movement model is determined by two parameters: the expected movement distance $d_{\max }$ between two position estimations and the weighting factor $w_{\text {move }}$ for the movement force $\vec{F}_{\text {move }}$. Figure 7 (right) shows the influence of $w_{\text {move }}$ for the force-directed approach without step detection $\left(d_{\max }=0.2 \mathrm{~m}\right)$ and with step detection $\left(d_{\max }=0.5 \mathrm{~m}\right)$. Note that normally one would choose the values of $d_{\max }$ a little higher, e.g., $d_{\max }=0.35 \mathrm{~m}$ per 0.25 seconds or $d_{\max }=0.65 \mathrm{~m}$ per step. We chose the values deliberately a little shorter to be able to demonstrate the influence of $w_{\text {move }}$.

If $w_{\text {move }}$ is chosen very low, the movement force plays almost no role for the final movement direction. In this case, we are back at the situation without movement forces, which was analyzed in the trilateration section. For high values of

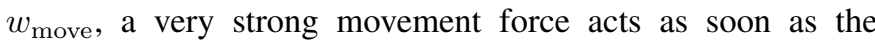
position estimate leaves the area with radius $d_{\max }$ around the last position estimate. But no matter how large $w_{\text {move }}$ is chosen, the position estimate still can move distance $d_{\text {max }}$ per step. This explains why the localization error in Figure 7 (right) does not increase significantly for high values of $w_{\text {move }}$.

From a practical perspective, the modeling with a radius $d_{\max }$ within which no movement forces act offers some advantages. There are many scenarios for which it is easy to define a meaningful value for $d_{\max }$. For example, a single step cannot be arbitrary wide. In contrast, in the analyzed EKF model the actual movement distance is determined by a combination of several complex factors, such as uncertainties of single measurements for example. This makes it much harder to model such kinds of knowledge. Of course, it can happen that $d_{\max }$ is chosen significantly too small for the considered application. In this case, the outlined force-directed approach behaves very similar to the EKF.

According to the curves in Figure 7 (right), the optimum values for $w_{\text {move }}$ are $w_{\text {move }}=385$ for the model without step recognition and $w_{\text {move }}=710$ for the model with step recognition. As for the EKF, we will use these optimal parameters in the following studies.

2) Influence of Number of Landmarks: Figure 8 shows the average error over all runs in dependance of the number of

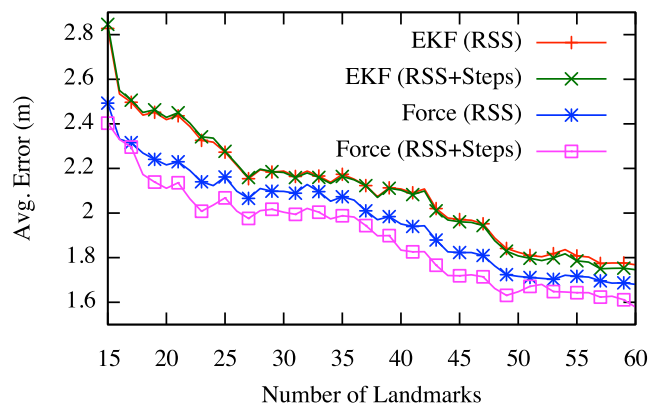

Fig. 8. Tracking: influence of number of landmarks.

landmarks. The used landmarks were selected as described in Section VI-C2. Again, for all approaches the 10 best RSS measurements were used. The picture is quite similar to the last paragraph, with the force-directed approaches showing a slightly smaller error than the EKF. Apparently, the EKFs with and without step detection show nearly the same average error. We assume that as long as the person walks somewhat steadily, it makes no big difference whether the position update is done with every measurement or every step, as long as the parameters are chosen accordingly. Also, it can be seen that depending on the intended application even a small network with only 15 to 20 landmarks might be sufficient to achieve a mean localization accuracy below $2.5 \mathrm{~m}$.

So far, all presented experimental results were averaged over all instances. Table II shows how the algorithms behave on single runs. The results were achieved using all 60 landmarks and the 10 best RSS measurements per time step.

\begin{tabular}{|l|c|c|c|c|c|c|c|c|c|}
\hline \multirow{2}{*}{ Algorithm / Scenario } & \multicolumn{4}{|c|}{ Building 1} & \multicolumn{5}{c|}{ Building 2} \\
\cline { 2 - 9 } & 1 & 2 & 3 & 4 & 1 & 2 & 3 & 4 & 5 \\
\hline Kalman (RSS) & 1.69 & 1.54 & 1.44 & 1.32 & 1.67 & 2.11 & 1.83 & 2.25 & 2.06 \\
Kalman (RSS+Steps) & 1.56 & 1.59 & 1.48 & 1.34 & 1.68 & 2.03 & 1.77 & 2.18 & 2.08 \\
Force (RSS) & 1.88 & 1.41 & 1.39 & 1.06 & 1.37 & 2.22 & 1.95 & 2.37 & 1.47 \\
Force (RSS+Steps) & 1.47 & 1.41 & 1.63 & 1.24 & 1.30 & 1.85 & 1.71 & 2.10 & 1.49 \\
\hline
\end{tabular}

TABLE II

AVERAGE LOCALIZATION ERROR IN METERS (TRACKING)

\section{E. Tracking Examples}

To conclude the experimental part, two actual tracking instances are presented in this section. The path estimate in Figure 9 was computed using the force-directed method without step detection. Figure 10 shows a localization result using

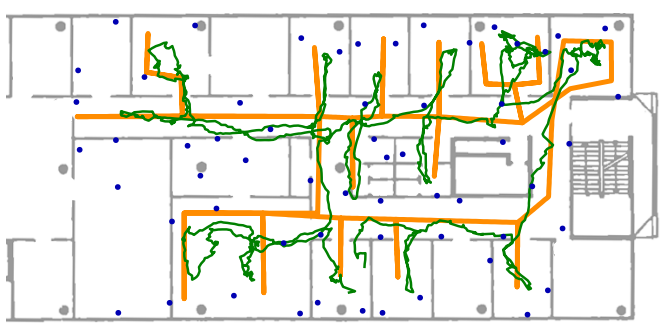

Fig. 9. Tracking results in building 1 using the force-directed approach without step recognition. The bold path shows the reference trajectory, the narrow path shows the computed trajectory. 
the force-directed method with step detection. We refrained from including corresponding EKF trajectories in the figures, as they would become confusing otherwise. Overall, the EKF trajectories look very similar.

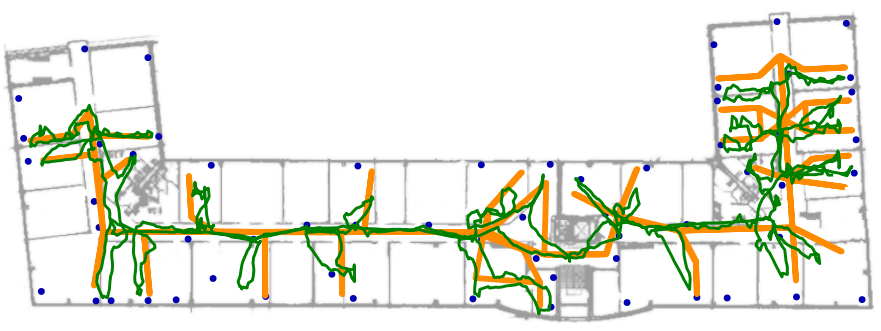

Fig. 10. Tracking results in building 2 using the force-directed approach with step recognition. The bold path shows the reference trajectory, the narrow path shows the computed trajectory.

\section{CONCLUSion AND Future Work}

We presented a first study on the application of forcedirected approaches to the tracking problem in wireless networks. To motivate design decisions and to evaluate the achievable performance, experimental data from a 60-node wireless sensor network was used. Additionally, variants of extended Kalman filters were implemented as reference approaches, and similarities and distinctions between both kinds of approaches were highlighted.

In our experiments, we considered the trilateration problem to identify adequate force definitions for the modeling of RSS-based distance estimations. Additionally, we analyzed the influence of the number of landmarks and the number of used measurements on the achievable trilateration accuracy. In our study of the tracking problem, we examined the parameter sensitivity of the considered approaches and the dependance of the tracking accuracy on the number of used landmarks. Our experimental results indicate that for the scenarios considered in this paper, force-directed approaches offer a competitive alternative to the widely used extended Kalman filters.

Force-directed tracking algorithms offer an immense freedom in building up a system model. In comparison to the EKF, process state and measurement inputs are not limited to normal distributions. Additionally, nonlinear measurements can be used directly without linearization. As runtime and space complexity of the outlined force-directed algorithm depend linearly on the number of measurements, it is well suited even for usage on hardware with high resource constraints.

This paper can only serve as a starting point and further research is necessary to investigate the possibilities forcedirected approaches offer in the context of tracking in wireless networks. For example, one could extend the model with information from inertial sensors like step length and velocity. Heading from an electronic compass and gyroscope readings could be used to gain additional information on the moving direction. Another idea is to use position estimates of forcedirected approaches as an additional input to Kalman filters, thus combining the strengths of both approaches.

\section{ACKNOWLEDGMENTS.}

This work was supported by the German Research Foundation (DFG) within the Research Training Group GRK 1194.

\section{REFERENCES}

[1] N. Patwari, J. Ash, S. Kyperountas, A. Hero III, R. Moses, and N. Correal, "Locating the Nodes: Cooperative Localization in Wireless Sensor Networks," Signal Processing Magazine, IEEE, vol. 22, no. 4, pp. 54-69, 2005.

[2] S. Park and S. Hashimoto, "Autonomous Mobile Robot Navigation using Passive RFID in Indoor Environment," Industrial Electronics, IEEE Transactions on, vol. 56, no. 7, pp. 2366-2373, 2009.

[3] V. Fox, J. Hightower, L. Liao, D. Schulz, and G. Borriello, "Bayesian Filtering for Location Estimation," Pervasive Computing, IEEE, vol. 2, no. 3, pp. 24-33, 2003.

[4] K. Lorincz and M. Welsh, "MoteTrack: a Robust, Decentralized Approach to RF-based Location Tracking," Personal and Ubiquitous Computing, vol. 11, no. 6, pp. 489-503, 2007.

[5] M. Azizyan and R. Choudhury, "SurroundSense: Mobile Phone Localization using Ambient Sound and Light," ACM SIGMOBILE Mobile Computing and Communications Review, vol. 13, no. 1, pp. 69-72, 2009.

[6] J. Park, B. Charrow, D. Curtis, J. Battat, E. Minkov, J. Hicks, S. Teller, and J. Ledlie, "Growing an Organic Indoor Location System," in Proceedings of the 8th International Conference on Mobile Systems, Applications, and Services. ACM, 2010, pp. 271-284.

[7] X. Li, "RSS-Based Location Estimation with Unknown Pathloss Model," Wireless Communications, IEEE Transactions on, vol. 5, no. 12, pp. 3626-3633, 2006.

[8] Pahlavan, K. and Akgul, F.O. and Heidari, M. and Hatami, A. and Elwell, J.M. and Tingley, R.D., "Indoor Geolocation in the Absence of Direct Path," Wireless Communications, IEEE, vol. 13, no. 6, pp. 50-58, 2006.

[9] A. Paul and E. Wan, "Rssi-Based Indoor Localization and Tracking using Sigma-Point Kalman Smoothers," Selected Topics in Signal Processing, IEEE Journal of, vol. 3, no. 5, pp. 860-873, 2009.

[10] J. Schmid, F. Beutler, B. Noack, U. Hanebeck, and K. Müller-Glaser, "An Experimental Evaluation of Position Estimation Methods for Person Localization in Wireless Sensor Networks," Wireless Sensor Networks, pp. 147-162, 2011.

[11] J. Schmid, D. Curtis, T. Gaedeke, and J. Ledlie, "Improving sparse organic wifi localization with inertial sensors," in Positioning, Navigation and Communication 2012 (WPNC'12), 9th Workshop on. IEEE, 2012.

[12] Y. Shang, W. Rumi, Y. Zhang, and M. Fromherz, "Localization from Connectivity in Sensor Networks," Parallel and Distributed Systems, IEEE Transactions on, vol. 15, no. 11, pp. 961-974, 2004.

[13] D. Moore, J. Leonard, D. Rus, and S. Teller, "Robust Distributed Network Localization with Noisy Range Measurements," in Proceedings of the 2nd International Conference on Embedded Networked Sensor Systems. ACM, 2004, pp. 50-61.

[14] A. Efrat, D. Forrester, A. Iyer, S. G. Kobourov, C. Erten, and O. Kilic, "Force-Directed Approaches to Sensor Localization," ACM Transactions on Sensor Networks (TOSN), vol. 7, pp. 27:1-27:25, 2010.

[15] K. Coogan, V. Khare, S. G. Kobourov, and B. Katz, "MSDR-D Network Localization Algorithm," in 6th International Conference on Algorithms for Sensor Systems, Wireless Adhoc Networks, and Autonomous Mobile Entities, 2010, pp. 148-160.

[16] P. Eades, "A Heuristic for Graph Drawing," Congressus Numerantium, vol. 42, pp. 149-160, 1984.

[17] T. M. J. Fruchterman and E. M. Reingold, "Graph Drawing by Forcedirected Placement," Software - Practice and Experience (SPE), vol. 21, no. 11, pp. 1129-1164, 1991.

[18] P. S. Maybeck, Stochastic Models, Estimation, and Control, ser. Mathematics in Science and Engineering. Academic Press, 1979, vol. 141.

[19] G. Welch and G. Bishop, "An Introduction to the Kalman Filter," Design, vol. 7, no. 1, pp. 1-16, 2001.

[20] G. Mao, B. Anderson, and B. Fidan, "Path Loss Exponent Estimation for Wireless Sensor Network Localization," Computer Networks, vol. 51 , no. 10, pp. 2467-2483, 2007.

[21] L. Liechty, "Path Loss Measurements and Model Analysis of a $2.4 \mathrm{GHz}$ Wireless Network in an Outdoor Environment," Master's thesis, Georgia Institute of Technology, 2007. 